Discussion/conclusion The curriculum competences-based approach to training evaluation offers a focused and objective approach to resolve conflict of training and service provision. Furthermore, it highlights and supports formalisation of nonclinical training opportunities.

\section{P204 SEX UNDRESSED: DEVELOPING A WEBSITE FOR YOUNG PEOPLE AROUND SEX AND BODY IMAGE}

${ }^{1}$ Alice Armitage ${ }^{*},{ }^{2}$ Cait Crosse, ${ }^{3}$ Alan Bridger. ${ }^{1} U C L$, London, UK; ${ }^{2}$ English for Action London, London, UK; ${ }^{3}$ Makers Academy, London, UK

\subsection{6/sextrans-2015-052126.248}

Introduction Evidence shows that children and young people (CYP) have inter-related issues around self-esteem, body image, sex and relationships. Access to pornography and paucity of sex and relationship education in the UK is exacerbating this problem.

We want to develop an online resource for CYP addressing sex and body image, centred around videos from 'peer educators' but also containing information and links to other resources.

Aims To undertake a consultation period with the aim of informing the content and aims of the website.

Methods Workshops comprising: 1. Introduction of website concept. 2. Card-cluster participatory exercise, 'what I wish I had known when I was younger'. 3. Feedback.

Results 3 workshops were held, (participants $=19$, M11, F8, age 20-30).

The concept was well-received and most participants wished to be involved. Concerns were expressed about people's willingness to speak openly about intimate issues in videos and potential for cultural bias in participation.

Card-cluster themes included rejecting the portrayal of sex in pornography, rejecting the portrayal of body image in the media, normalising masturbation, normalising body hair and concepts of consent and the right to enjoy sex.

$70 \%$ felt clear about the project, and 100\% felt included. Conclusion Our findings indicate strikingly similar concerns and insecurities that could be amenable to positive role models and educational resources in a youth-friendly website. We recognise the need to engage diverse groups (in terms of race, class, sexuality) to encourage inclusive content and users.

\section{Category: STIs in special groups}

\section{P205 RETESTING FOR GENITAL CHLAMYDIA INFECTIONS IN YOUNG PEOPLE IS ACCEPTABLE AND IMPORTANT}

${ }^{1}$ Adam Mclean*, ${ }^{2}$ Rebecca Metcalfe. ${ }^{1}$ University of Glasgow, Glasgow, UK; ${ }^{2}$ Sandyford Sexual Health Clinic, Glasgow, UK

\subsection{6/sextrans-2015-052126.249}

Background/introduction Young persons with a previous history of genital chlamydia are more likely to retest positive at a later date than those that have never tested positive. Current recommendations advise offering a repeat NAAT test to individuals under the age of 25 , three months after completing treatment. However, a recall system has yet to be implemented in our clinic.
Objectives We examined how many young people treated for genital chlamydia voluntarily returned for a further test, to assess whether asymptomatic retesting is both acceptable and worthwhile in this group. We aimed to identify a suitable timeframe in which retesting should be offered.

Methods Retrospective case note review of individuals under 25 years who tested positive for genital chlamydia in a city wide sexual health service in January 2013.

Results Of 214 individuals testing positive for genital chlamydia in January 2013, 50\% (107/214) retested within 15 months, $29 \%$ of which were positive (31/214). Most young people returned 3 to 6 months following their initial diagnosis (37/ 107), but the highest number of positive results occurred between 7 and 9 months (10/31). Only 8 individuals (7.5\%) were retested between 10 and 12 months, though a significant proportion (25\%) retested positive.

Conclusion The high rate of young people returning for chlamydia retesting after a positive diagnosis indicates that retesting is acceptable within this group. The high rate of subsequent positive tests suggests that retesting is important and worthwhile. The ideal timeframe to retest these individuals is $3-12$ months following a positive test.

\section{P206 SEXUAL HEALTH RISKS, SERVICE USE, AND VIEWS OF RAPID POINT-OF-CARE TESTING AMONG MEN-WHO- HAVE-SEX-WITH-MEN ATTENDING SAUNAS: A CROSS SECTIONAL SURVEY}

${ }^{1}$ Jeremy Horwood*, 'Suzanne Ingle, 'David Burton, 'Adam Woodman-Bailey, 1,2Paddy Horner, ${ }^{1,2}$ Nicola Jeal. 'University of Bristol, Bristol, UK; ${ }^{2}$ University Hospitals Bristol NHS Foundation Trust, Bristol, UK

\subsection{6/sextrans-2015-052126.250}

Background/introduction Guidelines highlight the need to increase HIV testing amongst men-who-have-sex-with-men (MSM) as a priority and recommend MSM at high risk of HIV test for every three months. Novel point of care testing (POCT) provides new possibilities for delivery of care. However, it is unclear how POCT should be used to best effect.

Aim(s)/objectives This study aimed to increase understanding of sexual risk-taking behaviour, service use and attitudes to POCT amongst sauna clients.

Methods Data were collected within two saunas for MSM in south west England using a self-completion survey on a computer tablet device.

Results 134 men participated (74\% response rate). Half of participants (51\%) reported unprotected anal intercourse (UAI) with a casual partner in the previous three months. For those reporting UAI, 19\% reported having an STI test and 16\% had taken an HIV test in the previous three months. Participants reported they would be more likely to be tested for HIV (84\%), gonorrhoea (91\%), chlamydia (90\%) and syphilis (90\%) if available as rapid POCT to avoid a stressful wait. The majority of men $(52 \%)$ would prefer to receive POCT at NHS sexual health clinics.

Discussion/conclusion Though this sample of sauna clients are at high risk of acquiring an STI, the testing frequency amongst the majority of those reporting UAI is not in keeping with national guidelines. For almost all participants the introduction of rapid POCT for both genital and blood-borne infection was likely to increase testing and for the majority NHS specialist services was the preferred setting. 